\title{
X-rays From Centrifugal Magnetospheres in Massive Stars
}

\author{
Christopher Bard and Richard Townsend \\ University of Wisconsin, Madison \\ corresponding email: bard@astro.wisc.edu
}

\begin{abstract}
In the subset of massive OB stars with strong global magnetic fields, X-rays arise from magnetically confined wind shocks (Babel \& Montmerle 1997). However, it is not yet clear what the effect of stellar rotation and mass-loss rate is on these wind shocks and resulting Xrays. Here, we present results from a grid of Arbitrary Rigid-Field Hydrodynamic simulations (ARFHD) of a B-star centrifugal magnetosphere with an eye towards quantifying the effect of stellar rotation and mass-loss rate on the level of X-ray emission. The results are also compared to a generalized XADM model for X-rays in dynamical magnetospheres (ud-Doula et al. 2014).
\end{abstract}

\section{Arbitrary Rigid-Field Hydrodynamic Simulations}

The strong magnetic fields of centrifugal magnetospheres produce Alfvén speeds so fast that MHD simulations are impractical. To address this issue, Townsend et al. (2007) developed a Rigid-Field Hydrodynamics (RFHD) approach to simulate magnetospheres in the limit of strong magnetic confinement. The three-dimensional stellar outflow is approximated as many quasi-one-dimensional flows along individual field lines subject to radiative and gravitocentrifugal forces. We simulate each line separately and stitch together the results to form a picture of the overall magnetosphere.

We have since extended the RFHD technique to incorporate completely arbitrary magnetic configurations (arbitrary RFHD, or ARFHD; Bard \& Townsend, in prep.), though all of the simulations presented here focus on the simplest case of a rotation-aligned dipole field. Our goal is to understand the effect of stellar rotation and mass-loss rate on the level of X-ray emission in a B-star centrifugal magnetosphere.

Towards this end, we simulate a star with an aligned dipole magnetic field $\left(\beta=0^{\circ}\right)$ and other fundamental parameters based on the archetype $\sigma$ Ori E (Townsend et al. 2013): $M=8.3 M_{\odot}, R=3.8 R_{\odot}, B=11 \mathrm{kG}$, and $T_{\text {eff }}=22500 \mathrm{~K}$. To understand the effect of rotation and mass-loss rate $(\dot{M})$ on the X-ray luminosity, we simulate every possible combination of six critical rotation fractions $W(0.0,0.2,0.35,0.5,0.65,0.8)$ and seven $\dot{M}$ values $\left(1 \cdot 10^{-5}, 1 \cdot 10^{-6}, 1 \cdot 10^{-7}, 1 \cdot 10^{-8}, 1 \cdot 10^{-9}, 1 \cdot 10^{-10}, 1 \cdot 10^{-11} M_{\odot} / \mathrm{yr}\right)$. We vary $\dot{M}$ through the $Q$ opacity parameter; see Gayley (1995) for a discussion.

\section{Results and Discussion}

In general, X-ray luminosity increases with both rotation and mass-loss rate (Fig 1). This is expected, since increasing the rotation rate provides a higher acceleration of the plasma along the field lines, resulting in a higher shock velocity and more X-ray emitting gas. Increasing the mass-loss rate increases the amount of density in the magnetosphere, which in turn increases the X-ray emission. Additionally, the results match up well with a semi-analytic XADM model (ud-Doula et al. 2014) calculated for a non-rotating dynamical magnetosphere (dashed line in Fig. 1). Although the XADM model was developed for dynamical magnetospheres, it applies well to our centrifugal magnetosphere simulations. 


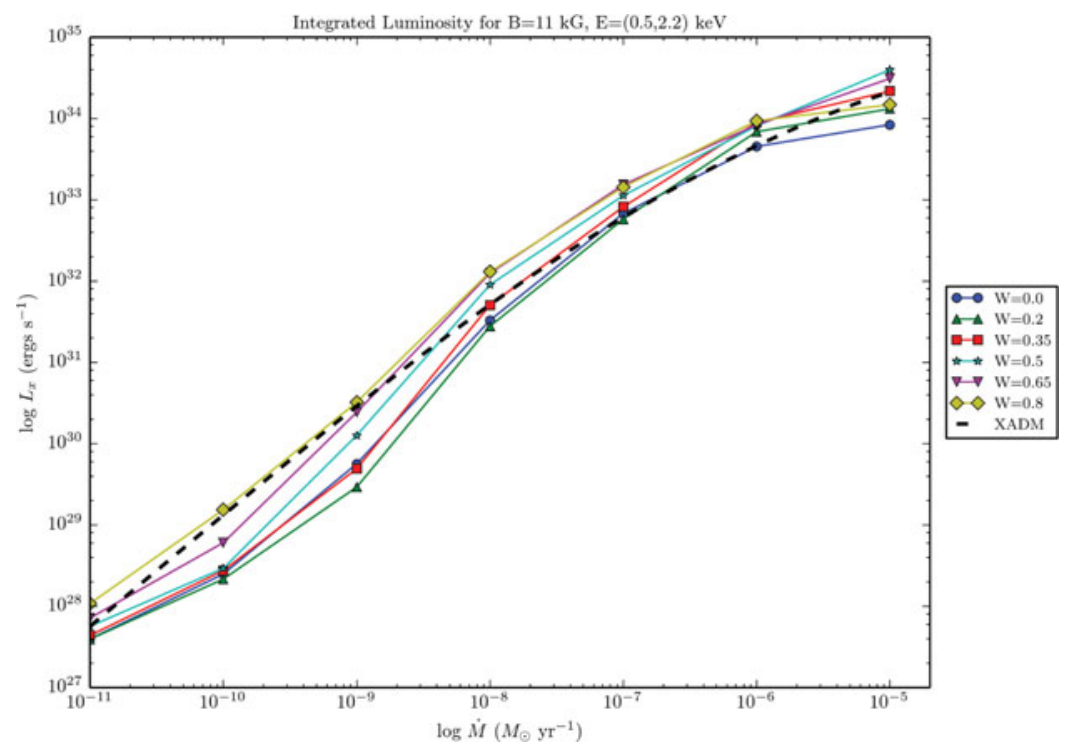

Figure 1. The relationship between integrated luminosity and mass-loss rate for varying critical rotation fraction. The dashed line is the predicted X-ray luminosity from a non-rotating XADM scaling law for $\mathrm{B}=11 \mathrm{kG}$. The results generally agree with the scaling law.

The XADM model does not include rotation, however. To better understand the dependence of X-ray luminosity on rotation rate, we fit our centrifugal magnetosphere simulation data as a power law $L_{x} \propto W^{\alpha}$ for each mass-loss rate. The average $\alpha$ is 0.88 , though there is a wide range $(\min =0.17, \max =1.84)$ among mass-loss rates. We believe this wide variation to be a result of siphon flows (e.g. Cargill \& Priest 1980) within our simulations. It is unclear whether these siphon flows are physical or simply a unphysical numerical solution for our quasi-1D field lines.

We derive a back-of-envelope $L_{x} \propto W^{\alpha}$ for comparison with our simulation power-law fit (with subscript 1 indicating pre-shock and 2 post-shock):

(a) In a strong shock, $T_{2} \propto v_{1}^{2}$.

(b) In a situation where only the rotation rate varies, we have $g_{\text {cen }} \propto W^{2}$, where $g_{\text {cen }}$ is the centrifugal acceleration along the field line.

(c) Making the simplifying assumption that $g_{\text {cen }}$ is independent of field line position, we get from kinematics that $v_{1}^{2} \propto g_{\text {cen }}$.

(d) X-ray emission in the magnetosphere is dominated by line emission: $L_{x} \propto T_{2}$.

From these relations, we naively expect $L_{x} \propto W^{2}$, but our simulation yields power-law coefficients smaller than 2 for every mass-loss rate. Future research will be needed to resolve this apparent discrepancy.

\section{References}

Babel, J. \& Montmerle, T. 1997, A\&A 323, 121

Cargill, P. J. \& Priest, E. R. 1980, Sol. Phys. 65, 251

Gayley, K. G. 1995, ApJ 454, 410

Townsend, R. H. D., Owocki, S. P., \& Ud-Doula, A. 2007, MNRAS 382, 139

Townsend, R. H. D., Rivinius, T., Rowe, J. F., et al. 2013, ApJ 769, 33

ud-Doula, A., Owocki, S., Townsend, R., Petit, V., \& Cohen, D. 2014, MNRAS 441, 3600 